\title{
Structure et dynamique de la végétation ligneuse juvénile issue de la régénération naturelle assistée (RNA) dans le Centre-Sud du Niger
}

\author{
Issoufou BAGGNIAN ${ }^{1 *}$, Toudou ADAM ${ }^{1}$, Mahaman Moustapha ADAMOU ${ }^{1}$, \\ Issa $\mathrm{CHAIBOU}^{3}$ et Ali MAHAMANE ${ }^{2,3}$ \\ ${ }^{1}$ Université Abdou Moumouni de Niamey, Faculté d'Agronomie, BP 10960, Niamey, Niger. \\ ${ }^{2}$ Université Abdou Moumouni de Niamey, Faculté des Sciences, Niamey, Niger. \\ ${ }^{3}$ Université de Maradi/Faculté des Sciences BP 465, Maradi, Niger. \\ *Auteur correspondant, E-mail : issoufou.baggnian@yahoo.fr
}

\section{RESUME}

La présente étude est conduite dans les régions de Maradi et Zinder situées dans la bande Sud du Niger où la pratique de la régénération naturelle assistée (RNA) a pris de l'ampleur depuis la fin des années 1980. Cette pratique qui consiste à entretenir les rejets a permis de régénérer le couvert végétal et d'augmenter le rendement des cultures pluviales. Le but de ce travail est d'étudier la structure et la dynamique de la végétation ligneuse juvénile issue de la régénération naturelle assistée. Tous les individus juvéniles dont le diamètre était inférieur à $4 \mathrm{~cm}$ ont été comptés dans des placettes de $2000 \mathrm{~m}^{2}$ suivant un transect perpendiculaire. L'importance écologique des espèces a été appréciée à partir de l'Indice de Valeur d'Importance (IVI). Les résultats révèlent que la flore juvénile de l'ensemble des sites renferme 38 espèces ligneuses réparties dans 22 familles. Les familles les mieux représentées sont les Mimosaceae (20,73\%). La strate juvénile dans l'ensemble a une dynamique déséquilibrée. En effet, très peu d'espèces ont une assez bonne rejuvénilisation. De nombreuses espèces quasi absentes de la population juvénile méritent de bénéficier d'une attention particulière afin de minimiser la perte de phytodiversité.

(ㅇ) 2014 International Formulae Group. All rights reserved.

Mots clés : Importance écologique, juvénile, flore, phytodiversité, rejuvénilisation

\section{INTRODUCTION}

$\mathrm{Au}$ cours des dernières décennies, la densité des arbres a considérablement diminué dans les paysages ruraux et les systèmes d'utilisation des terres des zones sahéliennes, soudaniennes et guinéennes, (Akpo et al., 2003). En effet, les fréquentes années de sécheresse survenues au Sahel entre 1960 et 1980 (Agnew et Chappell, 1999) ont provoqué la mortalité de certaines plantes ligneuses sur des vastes superficies (Toutain, 1999).

Ainsi, Reij et Botoni (2009) ont constaté que les formations naturelles ont largement disparu dans les régions à fortes densités de population humaine. Mais en revanche, il y a eu un développement significatif des systèmes agro-forestiers. C'est le cas des régions de Zinder et Maradi au 
Niger, la partie Nord du Plateau Central du Burkina Faso et la plaine du Gondo au Mali.

Plusieurs études qui ont porté sur des mesures du changement de la différence de l'indice de végétation normalisée (NDVI) saisonnier sur la base d'images satellitaires à haute résolution ont indiqué une augmentation de la végétation dans le Sahel depuis les années 1980 (Herrmann et al., 2005; Giannini, 2008). Le Sahel connaît sa plus grande transformation environnementale. Ainsi, plusieurs auteurs (Tougiani, 2009 ; Bégué et al., 2011) ont montré que dans de nombreuses régions du Sahel, la tendance à la dégradation des terres est contrée par un processus de «reverdissement» ou «ré-végétalisation».

Au Niger, diverses études menées dans les régions de Maradi et Zinder (Larwanou et Saadou, 2011; Haglund et al., 2011) ont prouvé que le reverdissement a lieu grâce aux efforts de réhabilitation effectués par différents acteurs, dont les agriculteurs, grâce à la pratique de la régénération naturelle assistée (RNA). Cette pratique a permis une augmentation de la densité d'arbres sur environ cinq millions d'hectares entre 1983 et 2005 dans la bande Sud du pays (Reij et Botoni, 2009).

Les études sur la régénération naturelle assistée des ligneux au Niger sont nombreuses. Ils ont porté sur l'ampleur, les facteurs favorables à l'adoption, l'impact de la pratique (Larwanou et al., 2012). Certaines de ces études ont abordé le rôle des interventions humaines sur la dynamique des arbres (Larwanou et Saadou, 2012). D'autres ont évalué la reconstruction de la résilience au Sahel: reverdissement dans les régions de Maradi et Zinder (Sendzimir et al., 2011) et les enjeux socio-économiques autour de l'agroforesterie villageoise (Dramé et Berti, 2008).

A ce jour, aucune étude ne s'est réellement intéressée spécifiquement à l'analyse démographique des peuplements juvéniles ligneux dans cette bande Sud. L'insuffisance de données actualisées et précises constitue une contrainte majeure. L'état démographique des jeunes plants reflète la dynamique des phytocénoses car il détermine le renouvellement des peuplements ligneux (Ouédraogo et al., 2006).

Dans la présente étude, il s'agit de mettre en évidence le rôle que jouent les jeunes plants dans l'établissement de la végétation adulte et de déterminer les stades de croissance où ces juvéniles présentent une grande vulnérabilité face aux facteurs de pression du milieu.

Elle contribuera, d'une part, à fournir des éléments indicateurs majeurs des tendances d'évolution qualitative et quantitative de la végétation des zones où le reverdissement a lieu et d'autre part, à mettre à la disposition des acteurs chargés de la mise en œuvre des politiques de gestion des ressources naturelles, des données fiables pouvant servir de guide pour l'adoption de choix ou de méthodes appropriées de conservation ou d'aménagement forestier pour une meilleure préservation des espèces végétales.

\section{MATERIEL ET METHODES}

\section{Sites d'étude}

L'étude s'est déroulée en 2012 dans deux terroirs villageois de la région de Maradi : Dan Saga situé à environ $20 \mathrm{~km}$ au Nord du chef-lieu de département d'Aguié, à $13^{\circ} 41^{\prime} 42,8^{\prime}$ ' latitude Nord et 7०44'11,6", longitude Est et El Guiéza situé à $17 \mathrm{~km}$ au Sud du chef-lieu du département d'Aguié. Il est déterminé par les coordonnées géographiques $13^{\circ} 25^{\prime} 58,3^{\prime}$ ' latitude Nord et 07'54'47,2', longitude Est. Puis dans trois terroirs de la région de Zinder: Daré situé à $19 \mathrm{~km}$ à l'Est de la commune urbaine de Matameye et à $115 \mathrm{~km}$ de Zinder, se localise aux coordonnées géographiques $13^{\circ} 19^{\prime}$ 08, $1^{\text {', }}$ de latitude Nord et $8^{\circ} 33^{\prime} 25,7^{\prime}$ ' de longitude 
Est ; Zedrawa situé à $2 \mathrm{~km}$ de Daré, se localise aux coordonnées géographiques 13²0' 14,3' 'de latitude Nord et 8 $8^{\circ} 33^{\prime} 16,4^{\prime \prime}$ de longitude Est et Ara Sofoua situé à environ 16 $\mathrm{km}$ au Nord de la ville de Magaria, chef-lieu de département. Ce village est localisé entre $13^{\circ} 08^{\prime} \quad 26,1 \quad 3$ ' de latitude Nord et $8^{\circ} 56^{\prime} 48,4$ '’ de longitude Est (Figure 1). Le choix des villages a été basé sur (i) la densité des arbres dans les champs relevée par des études antérieures (Dramé et Berti, 2008 ; Reij et Botoni, 2009) et (ii) l'existence de comités de surveillance de la RNA. La zone d'étude est relativement peuplée (environ 100 habitants $/ \mathrm{km}^{2}$ ). Le climat est semi-aride, caractérisé par un modèle unimodal de précipitation, avec une saison de pluies (de juin à septembre) et une saison sèche (d'octobre à mai). L'agriculture pluviale constitue près de $90 \%$ des activités de la population. Les céréales comme le mil et le sorgho sont principalement cultivés pour la subsistance sur des sols sableux et pauvres, dépendant entièrement des pluies.

\section{Caractérisation de la structure de la} végétation ligneuse juvénile

L'approche méthodologique est basée sur une analyse quantitative de la distribution des arbres dans les champs de culture. Pour ce faire, la méthode d'inventaire adoptée par Dramé et Berti, 2008 a été appliquée. L'inventaire des espèces ligneuses juvéniles a été effectué suivant les transects dans les 4 directions géographiques de chaque terroir villageois sur des placettes de $2000 \mathrm{~m}^{2}$ de superficie $(40 \mathrm{~m}$ x $50 \mathrm{~m})$ distantes de $500 \mathrm{~m}$. Tous les individus dont le diamètre est inférieur à $4 \mathrm{~cm}$ sont considérés comme la strate juvénile et ont été systématiquement considérés comme faisant partie de la régénération (Mahamane et Saadou, 2008). Au total, 103 placettes (23 placettes à Dan Saga, 21 à El Guiéza, 11 à Daré, 16 à Zedrawa et 32 à Ara Sofoua) ont été relevées.
À l'intérieur de chaque placette, les rejets et les plants issus de la germination des semences sont comptés par espèce et rangés dans cinq classes de hauteur d'intervalle 0,5 cm: [0-0,5 m [, [0,5-1 m[, [1-1,5 m[, [1,5-2 $\mathrm{m}[,[\geq 2 \mathrm{~m}]$. Le seuil de mesure suivant la hauteur et le diamètre des individus permettant de séparer la végétation juvénile de la végétation adulte reste très variable d'une étude à l'autre (Ouédraogo et al., 2009). Les mesures ont été effectuées pendant la saison des pluies (juin à juillet).

\section{Analyse et traitement des données}

L'importance écologique des espèces a été appréciée à partir de l'Indice de Valeur d'Importance (IVI) qui permet de mieux apprécier leur importance dans une communauté végétale (Traoré et al., 2011). Cet indice, pour une espèce, se définit comme la somme de sa dominance relative, de sa densité relative et de sa fréquence relative qui se calculent comme suit :

Dominance relative $=\frac{\text { ST Totale } \mathrm{B}}{\text { ST toutes } \mathrm{E}} \times 100$

où

ST Totale $\mathrm{E}=$ Surface terrière totale de l'espèce ST Toutes E=Surface terrière de toutes les espèces

$$
\mathrm{Fr}=\frac{\mathrm{F} \text { d'une espèce }}{\sum \mathrm{F} \text { des Espèces }} \times 100
$$

où $\mathrm{Fr}=$ Fréquence relative

$$
\mathrm{F}=\text { Fréquence }
$$

$\mathrm{Dr}=\frac{\mathrm{N} \text { d'individus de l'espèce / ha }}{\mathrm{N} \text { total d'individus / ha }} \times 100$

où $\mathrm{Dr}=$ Densité relative

$\mathrm{N}=$ Nombre

\section{Analyse de la structure démographique des espèces ligneuses juvéniles dominantes}

Les espèces à IVI $\geq 10$ sont celles écologiquement importantes (Reistma, 1988). Sur cette base, la structure démographique de la population juvénile dominante a été déterminée. 

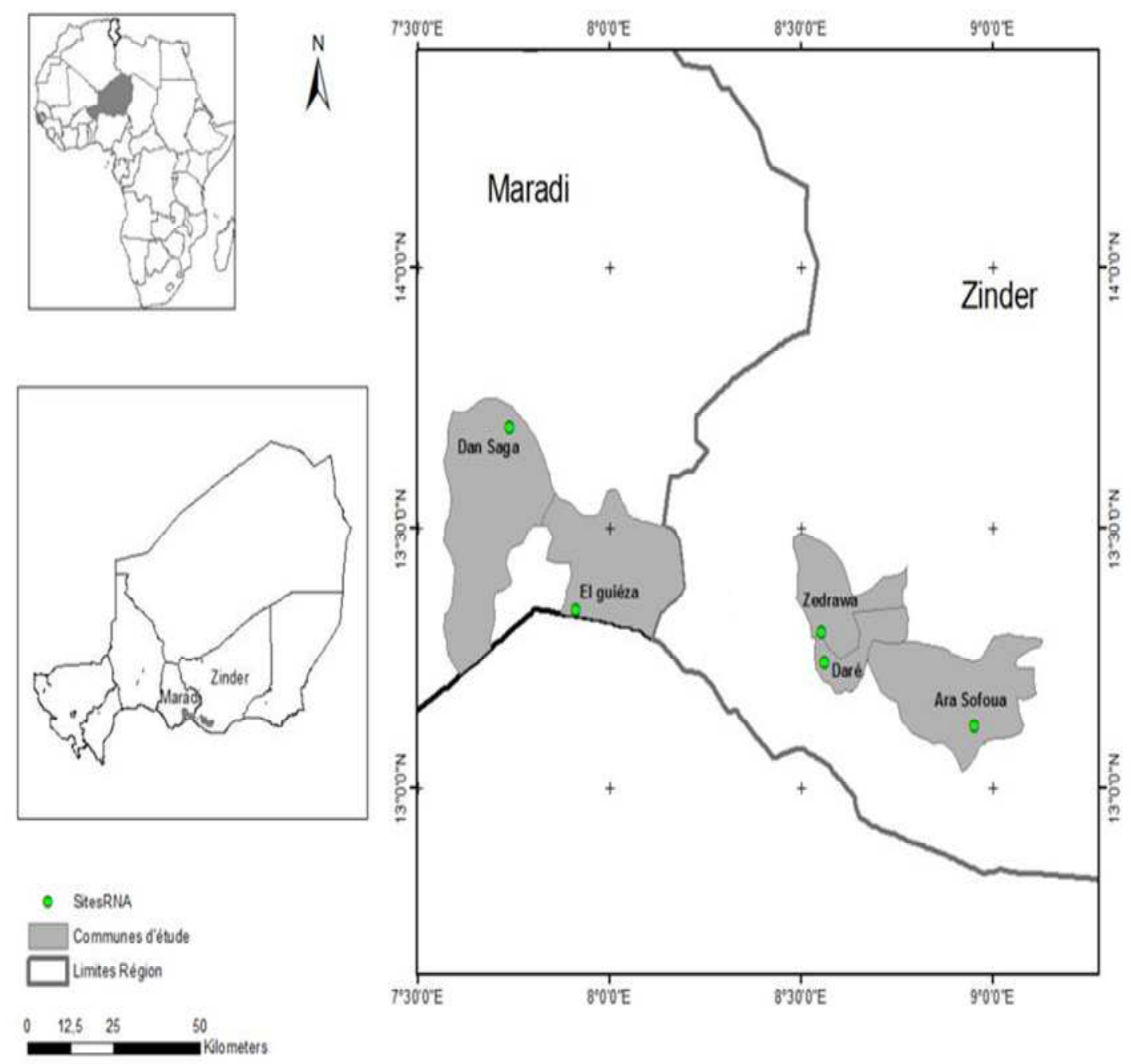

Figure 1 : Carte de localisation des sites d'études.

\section{RESULTATS}

Analyse de la composition floristique des espèces ligneuses juvéniles

La flore juvénile issue de l'ensemble des sites renferme 38 espèces ligneuses appartenant à 22 familles. Les familles les mieux représentées sont les Mimosaceae (20,73\%), suivies des Cesalpinaceae, Combretaceae $(9,76 \%)$ et des Anacardiaceae $(7,32)$; Rhamnaceae $(6,10 \%)$. Les espèces ligneuses juvéniles les plus abondantes sont
Guiera senegalensis $(26,26 \%)$ et Combretum glutinosum 18,72\% à Dan Saga; Hyphaene thebaica $(66,03)$ et Piliostigma reticulatum $(7,94 \%)$ à El Guiéza; Annona senegalensis (26,67\%) et Azadirachta indica $(22,22 \%)$ à Daré; Annona senegalensis $(46,32 \%)$ et Faidherbia albida $(29,47 \%)$ à Zedrawa ; Faidherbia albida (28,73\%) et Piliostigma reticulatum $(21,45 \%)$ à Ara Sofoua (Tableau 1). 
Structure juvénile globale selon les terroirs villageois et tendance démographique des espèces dominantes

Le comptage des juvéniles dans l'ensemble des sites a permis de dénombrer 1238 jeunes plants sur une superficie totale de 20,6 hectares (Figure 2).

\section{Dan Saga et El Guiéza ; région de Maradi}

Dans la région de Maradi, l'indice de valeur d'importance des espèces ligneuses juvéniles est plus élevé pour des espèces comme Guiera senegalensis $(65,19)$; Combretum glutinosum $(48,82)$ et Piliostigma reticulatum $(41,96 \%)$ dans la partie Nord (Dan Saga). Par contre, dans la partie Sud (El Guiéza), c'est Hyphaene thebaica $(154,76 \%)$; Piliostigma reticulatum $(40,68)$ et Ziziphus mauritiana $(22,85 \%)$ qui dominent (Tableau $1)$.

Cependant, le taux de survie est très faible, occasionnant une chute du taux de jeunes plants entre la strate $[0-0,5 \mathrm{~m}$ [ et [0,5$1 \mathrm{~m}$ [ aussi bien à Dan Saga qu' à El Guiéza (Figure 2). Plus de $30 \%$ des juvéniles n'arrivent pas à s'affranchir de la première strate qui marque la phase d'établissement de la régénération. Ce faible effectif dans ces strates traduit la vulnérabilité des nouvelles pousses et des jeunes plants de plus petite taille à la sècheresse et à la pression anthropique.

Les deux espèces dominantes à Dan saga Guiera senegalensis et Combretum glutinosum montrent des signes de mauvais état (Figure 3a). En témoigne la perte d'individus de la strate [0,5-1m [. En effet, les jeunes plants font l'objet d'exploitations diverses telles que la coupe de la fibre pour le tissage et la récolte des feuilles pour l'alimentation du petit ruminant en début de saison de pluie.

A El Guiéza, le potentiel en juvéniles est concentré dans la strate 3 en particulier. Mais il se produit une mortalité très importante des plants pendant la transition de la strate 1 à la strate 2 et de la strate 3 à la strate 4 (Figure 3b). $\mathrm{La}$ structure démographique de Hyphaene thebaica et Piliostigma reticulatum montre une vulnérabilité des juvéniles. Il y a une chute importante du nombre de juvéniles entre les deux premières strates (Figure $3 b$ ) et une absence de juvéniles dans les dernières strates.

\section{Daré, Zedrawa et Ara Sofoua (Centre-Sud de Zinder) \\ Dans le Centre-Sud de Zinder, l'indice} de valeur d'importance des espèces ligneuses juvéniles est plus élevé pour Annona senegalensis $(82,43 \%)$; il est suivi de Faidherbia albida (76,55\%) (Tableau 1).

La structure démographique des espèces juvéniles montre que le taux de survie est faible à Daré et Zédrawa et élevé à Ara Sofoua (Figure 2). En effet, à Daré et Zédrawa, le passage de la strate 1 à la strate 2 se fait avec un recrutement de plus de 50\% des jeunes individus. Mais ce taux chute à environ $30 \%$ dans la classe 3 . Cette forte chute démographique peut s'expliquer principalement par l'effet combiné des coupes et de la sècheresse qui perturbent la croissance et causent la mortalité de jeunes plants.

Structure du peuplement juvénile des espèces dominantes montre que seul Faidherbia albida présente une bonne dynamique dans la régénération à Daré. En revanche, Azadirachta indica et Eucalyptus camaldulensis a une structure déséquilibrée à cause du nombre élevé des individus de la dernière classe de régénération (Figure $3 \mathrm{c}$ ).

A Zedrawa, Annona senegalensis, Faidherbia albida et Commiphora africana semblent présenter une structure stable, sauf que l'absence des juvéniles dans la première strate de la régénération fait que la dynamique est perturbée (Figure 3d). Guiera senegalensis a une dynamique compromise par l'absence notoire des juvéniles dans presque toutes les strates. Enfin, à Ara Sofoua, les populations de Faidherbia albida sont en déséquilibre car les juvéniles des strates 2 et 3 sont moins nombreux que ceux des 2 dernières strates (Figure 3e). 
I. BAGGNIAN et al. / Int. J. Biol. Chem. Sci. 8(2): 649-665, 2014

Tableau 1 : Indice de valeur d'importance des espèces ligneuses juvéniles enregistrées dans les sites.

\begin{tabular}{|c|c|c|c|c|c|c|}
\hline Espèces & Nom local (Haoussa) & Familles & $\begin{array}{c}\text { Dominance } \\
\text { relative }\end{array}$ & $\begin{array}{c}\text { Fréquence } \\
\text { relative }\end{array}$ & $\begin{array}{l}\text { Densité } \\
\text { relative }\end{array}$ & IVI \\
\hline \multicolumn{7}{|c|}{ Région de Maradi } \\
\hline \multicolumn{7}{|c|}{ Dan Saga } \\
\hline Acacia nilotica (L.) Willd. ex Del. Subsp. & Bagaroua & Mimosaceae & 0,57 & 2,48 & 0,91 & 3,96 \\
\hline Acacia senegal (L.) & Ak kora & Mimosaceae & 0,28 & 0,83 & 0,23 & 1,34 \\
\hline Annona senegalensis Pers. & Gwada & Annonaceae & 13,38 & 9,92 & 12,79 & 36,09 \\
\hline Azadirachta indica A. Juss. & Bedi & Meliaceae & 2,78 & 4,13 & 1,60 & 8,51 \\
\hline Balanites aegyptiaca (L.) Del. & Adoua & Balanitaceae & 4,31 & 4,96 & 3,65 & 12,92 \\
\hline Bauhinia rufescens Lam. & Dirga & Cesalpinaceae & 0,01 & 1,65 & 0,46 & 2,12 \\
\hline Boscia salicifolia Oliv. & Zouré & Capparidaceae & 2,57 & 4,13 & 0,68 & 7,38 \\
\hline Boscia senegalensis (Pers.) Lam. ex Poir. & Anza & Capparaceae & 1,03 & 1,65 & 1,37 & 4,06 \\
\hline Calotropis procera (Ait.) Ait. f. & Tounfafia & Asclepiadaceae & 3,44 & 5,79 & 4,11 & 13,34 \\
\hline Cassia singuena (Del.) Lack. & Runhou & Cesalpinaceae & 3,05 & 8,26 & 4,79 & 16,11 \\
\hline Combretum glutinosum Perr. & Taramnia & Combretaceae & 19,35 & 10,74 & 18,72 & 48,82 \\
\hline Commiphora africana (A. Rich.) Engle. & Hiskitchi & Burseraceae & 1,10 & 3,31 & 1,14 & 5,55 \\
\hline Faidherbia albida Del. Chev. & Gao & Mimosaceae & 1,92 & 4,96 & 3,42 & 10,30 \\
\hline Guiera senegalensis J.F. Gmel. & Sabara & Combretaceae & 24,88 & 14,05 & 26,26 & 65,19 \\
\hline Hyphaene thebaica (L.) Mart. & Goriba/caba & Arecaceae & 0,23 & 1,65 & 0,91 & 2,79 \\
\hline Lannea microcarpa Engl. \& K. Krause & Farou & Anacardiaceae & 0,84 & 0,83 & 0,23 & 1,89 \\
\hline Maerua angolensis DC. & Tchi tchi wa & Capparaceae & 0,15 & 0,83 & 0,23 & 1,20 \\
\hline Maerua crassifolia Forsk. & Jiga & Capparaceae & 0,49 & 1,65 & 0,46 & 2,60 \\
\hline Moringa oleifera Lam. & Zogala & Moringaceae & 0,02 & 0,83 & 0,23 & 1,08 \\
\hline
\end{tabular}

654 
I. BAGGNIAN et al. / Int. J. Biol. Chem. Sci. 8(2): 649-665, 2014

\begin{tabular}{|c|c|c|c|c|c|c|}
\hline Piliostigma reticulatum (DC) Hachst. & Kalgo & Cesalpinaceae & 17,66 & 9,92 & 14,38 & 41,96 \\
\hline Prosopis africana (Guill et Perr) Lourb. & Kirya & Mimosaceae & 0,21 & 0,83 & 0,23 & 1,26 \\
\hline Slerocarya birrea (A. Rich.) Hachst. & Dania & Anacardiaceae & 0,42 & 1,65 & 0,46 & 2,53 \\
\hline Stereospermum kunthianum Cham. & Samsami & Bignoniaceae & 0,33 & 1,65 & 0,68 & 2,67 \\
\hline Ziziphus mauritiana Lam. & Magaria & Rhamnaceae & 0,96 & 3,31 & 2,05 & 6,32 \\
\hline \multicolumn{7}{|c|}{ El Guiéza } \\
\hline Albizia chevalerie Harms. & Katsari & Mimosaceae & 4,04 & 6,45 & 1,90 & 12,39 \\
\hline Annona senegalensis Pers. & Gwada & Annonaceae & 3,64 & 6,45 & 2,22 & 12,31 \\
\hline Balanites aegyptiaca (L.) Del. & Adoua & Balanitaceae & 1,20 & 3,23 & 0,63 & 5,06 \\
\hline Bauhinia rufescens Lam. & Dirga & Cesalpinaceae & 2,23 & 1,61 & 0,95 & 4,80 \\
\hline Calotropis procera (Ait.) Ait. f. & Tounfafia & Asclepiadaceae & 0,90 & 1,61 & 0,32 & 2,83 \\
\hline Cassia singuena (Del.) Lack. & Runhou & Cesalpinaceae & 0,39 & 3,23 & 2,54 & 6,16 \\
\hline Combretum glutinosum Perr. & Taramnia & Combretaceae & 0,51 & 1,61 & 0,32 & 2,44 \\
\hline Combretum micranthum G. don & Guéza & Combretaceae & 0,69 & 1,61 & 0,32 & 2,62 \\
\hline Commiphora africana (A. Rich.) Engle. & Hiskitchi & Burseraceae & 6,16 & 8,06 & 2,86 & 17,08 \\
\hline Dichrostachys cinerea & Doundou & Mimosaceae & 0,35 & 1,61 & 0,32 & 2,28 \\
\hline Diospyros mespiliformis Hochst. ex A. Rich. & Kangna & Ebenaceae & 0,69 & 1,61 & 0,32 & 2,62 \\
\hline Guiera senegalensis J.F. Gmel. & Sabara & Combretaceae & 0,23 & 1,61 & 0,32 & 2,16 \\
\hline Hyphaene thebaica (L.) Mart. & Goriba/caba & Arecaceae & 59,69 & 29,03 & 66,03 & 154,76 \\
\hline Maerua angolensis DC. & Tchi tchi wa & Capparaceae & 0,25 & 3,23 & 0,63 & 4,11 \\
\hline Piliostigma reticulatum (DC) Hachst. & Kalgo & Cesalpinaceae & 13,39 & 19,35 & 7,94 & 40,68 \\
\hline Prosopis africana (Guill et Perr) Lourb. & Kirya & Mimosaceae & 0,29 & 1,61 & 0,32 & 2,22 \\
\hline Securidaca longipedunculata Fres. & Ouwan magoun goun na & Polygalaceae & 0,69 & 1,61 & 0,32 & 2,62 \\
\hline Ziziphus mauritiana Lam. & Magaria & Rhamnaceae & 4,66 & 6,45 & 11,75 & 22,85 \\
\hline
\end{tabular}


I. BAGGNIAN et al. / Int. J. Biol. Chem. Sci. 8(2): 649-665, 2014

\begin{tabular}{|c|c|c|c|c|c|c|}
\hline \multicolumn{7}{|c|}{ Région de Zinder } \\
\hline \multicolumn{7}{|c|}{ Daré } \\
\hline Annona senegalensis Pers. & Gwada & Mimosaceae & 17,89 & 29,41 & 26,67 & 73,96 \\
\hline Azadirachta indica A. Juss. & Bedi & Meliaceae & 38,57 & 2,94 & 22,22 & 63,73 \\
\hline Cassia singuena (Del.) Lack. & Runfou & Cesalpinaceae & 0,15 & 2,94 & 1,11 & 4,20 \\
\hline Eucalyptus camaldulensis Dehnh. & Touraré & Myrtaceae & 20,30 & 2,94 & 14,44 & 37,68 \\
\hline Faidherbia albida Del. Chev. & Gao & Mimosaceae & 10,19 & 20,59 & 13,33 & 44,11 \\
\hline Guiera senegalensis J.F. Gmel. & Sabara & Combretaceae & 0,19 & 5,88 & 2,22 & 8,29 \\
\hline Lannea microcarpa Engl. \& K. Krause & Farou & Anacardiaceae & 2,17 & 5,88 & 2,22 & 10,27 \\
\hline Leptadenia hastata (Pers.) Decne. & Yadia & Asclepiadaceae & 2,05 & 11,76 & 11,11 & 24,93 \\
\hline Prosopis africana (Guill et Perr) Lourb. & Kirya & Mimosaceae & 5,97 & 5,88 & 2,22 & 14,08 \\
\hline Slerocarya birrea (A. Rich.) Hachst. & Dania & Anacardiaceae & 0,34 & 2,94 & 1,11 & 4,39 \\
\hline Ziziphus spina Christi Lam. & Kurna & Rhamnaceae & 0,36 & 5,88 & 2,22 & 8,47 \\
\hline Ziziphus mauritiana Lam. & Magaria & Rhamnaceae & 1,83 & 2,94 & 1,11 & 5,88 \\
\hline \multicolumn{7}{|c|}{ Zedrawa } \\
\hline Annona senegalensis Pers. & Gwada & Mimosaceae & 44,08 & 33,33 & 46,32 & 123,72 \\
\hline Balanites aegyptiaca (L.) Del. & Adoua & Balanitaceae & 1,38 & 2,78 & 1,05 & 5,21 \\
\hline Commiphora africana (A. Rich.) Engle. & Hiskitchi & Burseraceae & 18,29 & 11,11 & 10,53 & 39,93 \\
\hline Faidherbia albida Del. Chev. & Gao & Mimosaceae & 30,04 & 30,56 & 29,47 & $\mathbf{9 0 , 0 7}$ \\
\hline Guiera senegalensis J.F. Gmel. & Sabara & Combretaceae & 4,87 & 8,33 & 3,16 & 16,36 \\
\hline Leptadenia hastata (Pers.) Decne. & Yadiya & Asclepiadaceae & 0,98 & 8,33 & 7,37 & 16,69 \\
\hline Prosopis africana (Guill et Perr) Lourb. & Kirya & Mimosaceae & 0,25 & 2,78 & 1,05 & 4,08 \\
\hline Slerocarya birrea (A. Rich.) Hachst. & Dania & Anacardiaceae & 0,11 & 2,78 & 1,05 & 3,94 \\
\hline
\end{tabular}


I. BAGGNIAN et al. / Int. J. Biol. Chem. Sci. 8(2): 649-665, 2014

\begin{tabular}{|c|c|c|c|c|c|c|}
\hline \multicolumn{7}{|c|}{ Ara Sofoua } \\
\hline Acacia nilotica (L.) Willd. ex Del. Subsp. & Bagaroua & Mimosaceae & 0,48 & 0,95 & 0,36 & 1,80 \\
\hline Acacia seyal Del. & Fara kaya & Mimosaceae & 0,48 & 0,95 & 0,36 & 1,80 \\
\hline Adansonia digitata $\mathrm{L}$. & Kouka & Bombacaceae & 1,51 & 0,95 & 0,36 & 2,83 \\
\hline Annona senegalensis Pers. & Gwada & Annonaceae & 13,84 & 19,05 & 16,73 & 49,62 \\
\hline Azadirachta indica A. Juss. & Bedi & Meliaceae & 0,16 & 0,95 & 0,36 & 1,47 \\
\hline Balanites aegyptiaca (L.) Del. & Adoua & Balanitaceae & 0,63 & 0,95 & 0,36 & 1,94 \\
\hline Calotropis procera (Ait.) Ait.f. & Tounfafia & Asclepiadaceae & 0,75 & 2,86 & 1,09 & 4,70 \\
\hline Commiphora africana (A. Rich.) Engle. & Hiskitchi & Burseraceae & 2,69 & 3,81 & 3,64 & 10,14 \\
\hline Faidherbia albida Del. Chev. & Gao & Mimosaceae & 41,04 & 25,71 & 28,73 & 95,48 \\
\hline Ficus platyphylla Del. & Gamji & Moraceae & 0,74 & 0,95 & 1,45 & 3,14 \\
\hline Guiera senegalensis J.F. Gmel. & Sabara & Combretaceae & 8,88 & 10,48 & 14,18 & 33,54 \\
\hline Leptadenia hastata (Pers.) Decne. & Yadiya & Asclepiadaceae & 1,91 & 0,95 & 3,27 & 6,14 \\
\hline Leptadenia pyrotechnica (Forsk.) Decne & Kalumbo & Asclepiadaceae & 0,40 & 1,90 & 0,73 & 3,03 \\
\hline Moringa oleifera Lam. & Zogala & Moringaceae & 0,04 & 0,95 & 0,36 & 1,36 \\
\hline Piliostigma reticulatum (DC) Hachst. & Kalgo & Cesalpinaceae & 23,16 & 18,10 & 21,45 & 62,71 \\
\hline Prosopis africana (Guill et Perr) Lourb. & Kirya & Mimosaceae & 1,15 & 3,81 & 2,91 & 7,87 \\
\hline Slerocarya birrea (A. Rich.) Hachst. & Dania & Anacardiaceae & 1,31 & 3,81 & 2,18 & 7,30 \\
\hline Strychnos spinosa Lam. & Kokiya & Loganiaceae & 0,25 & 0,95 & 0,36 & 1,56 \\
\hline Vitex doniana Sweet. & Doumnia & Verbenaceae & 0,25 & 0,95 & 0,36 & 1,56 \\
\hline Ziziphus spina Christi Lam. & Kurna & Rhamnaceae & 0,33 & 0,95 & 0,73 & 2,01 \\
\hline
\end{tabular}




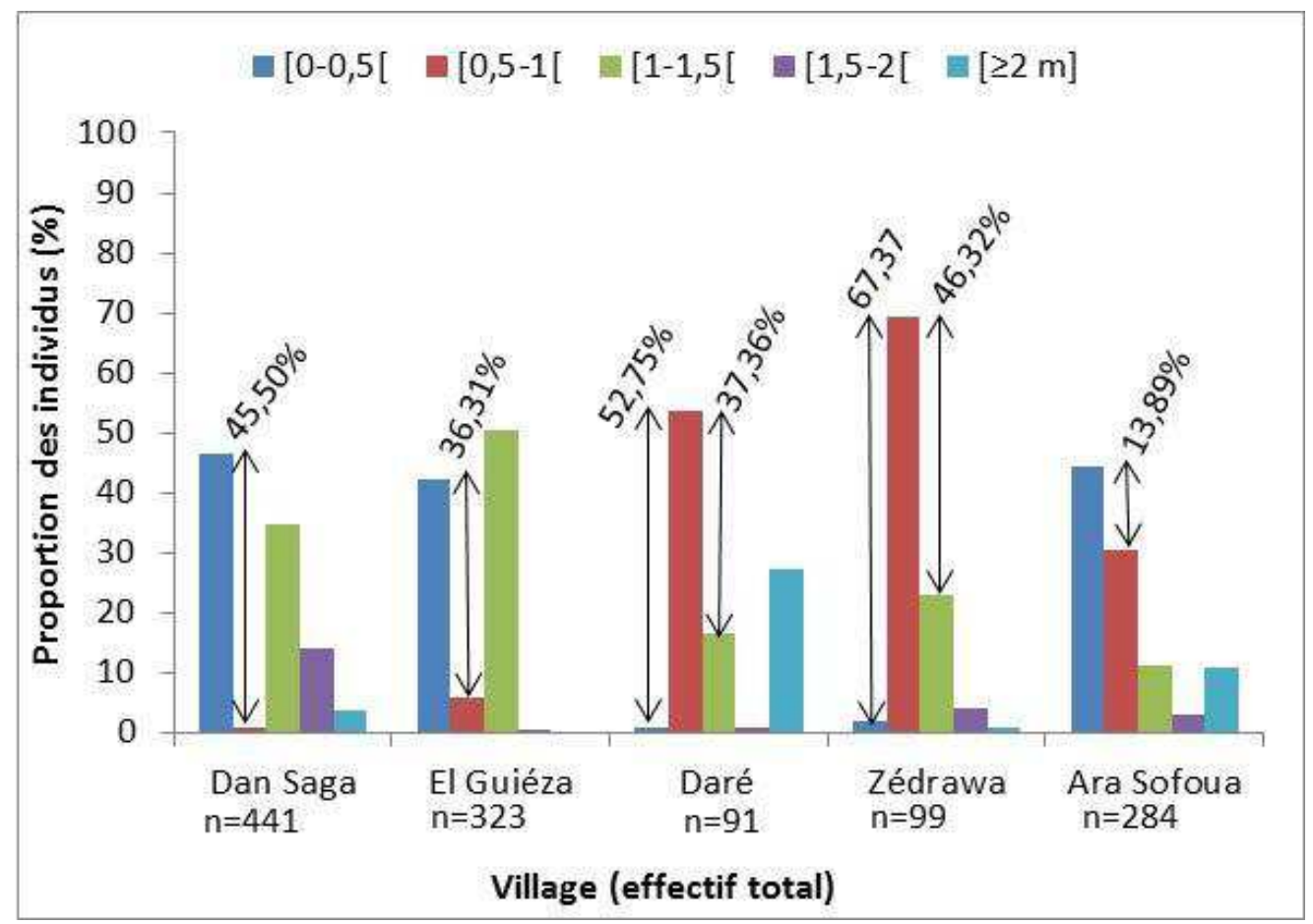

Figure 2: Structure de la population juvénile globale en classes de hauteur dans les sites inventoriés. n: nombre d'individus.

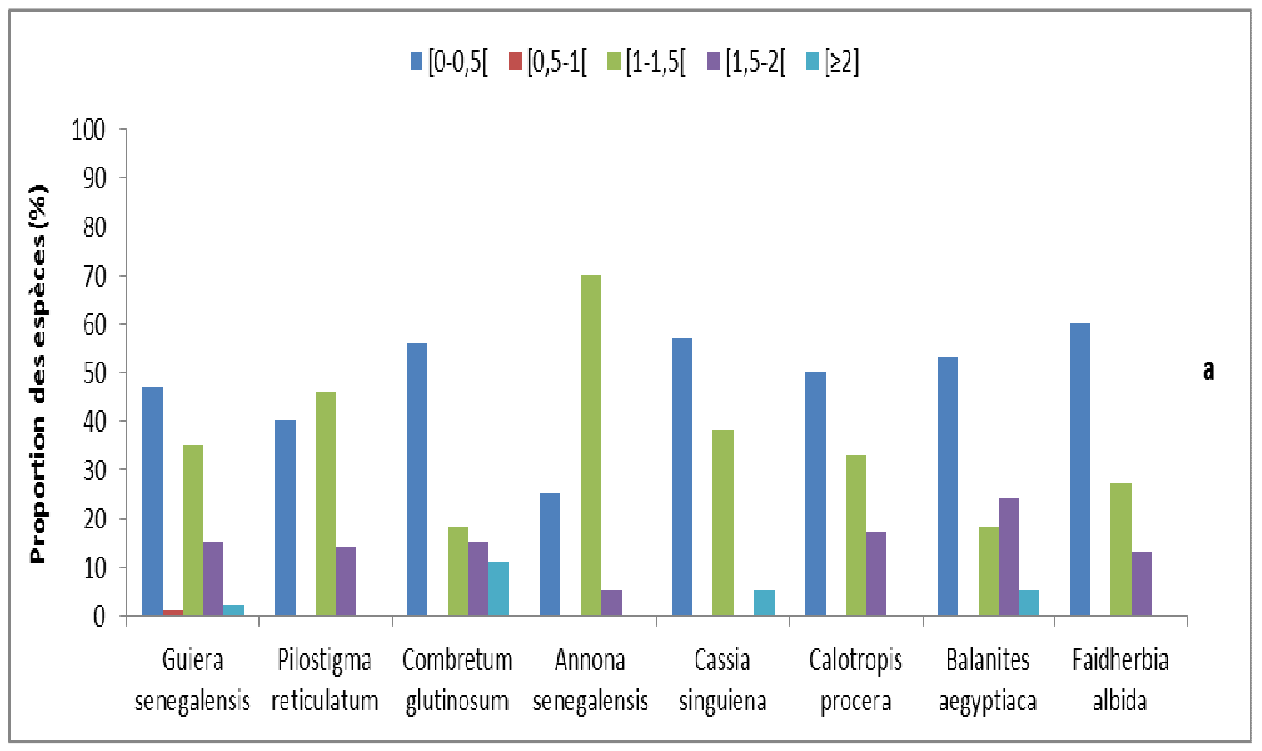

Figure 3a : Structure du peuplement juvénile des espèces dominantes à Dan Saga. 


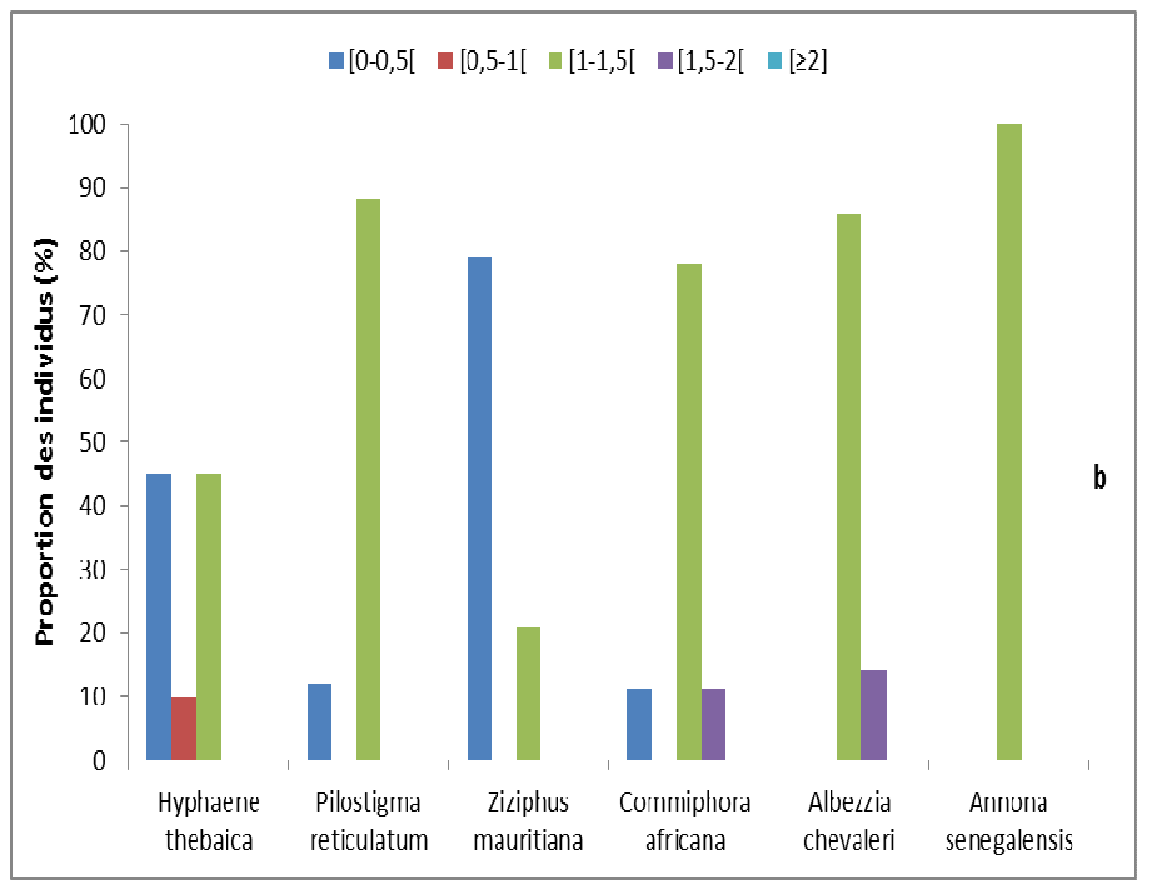

Figure 3b : Structure du peuplement juvénile des espèces dominantes à El Guiéza.

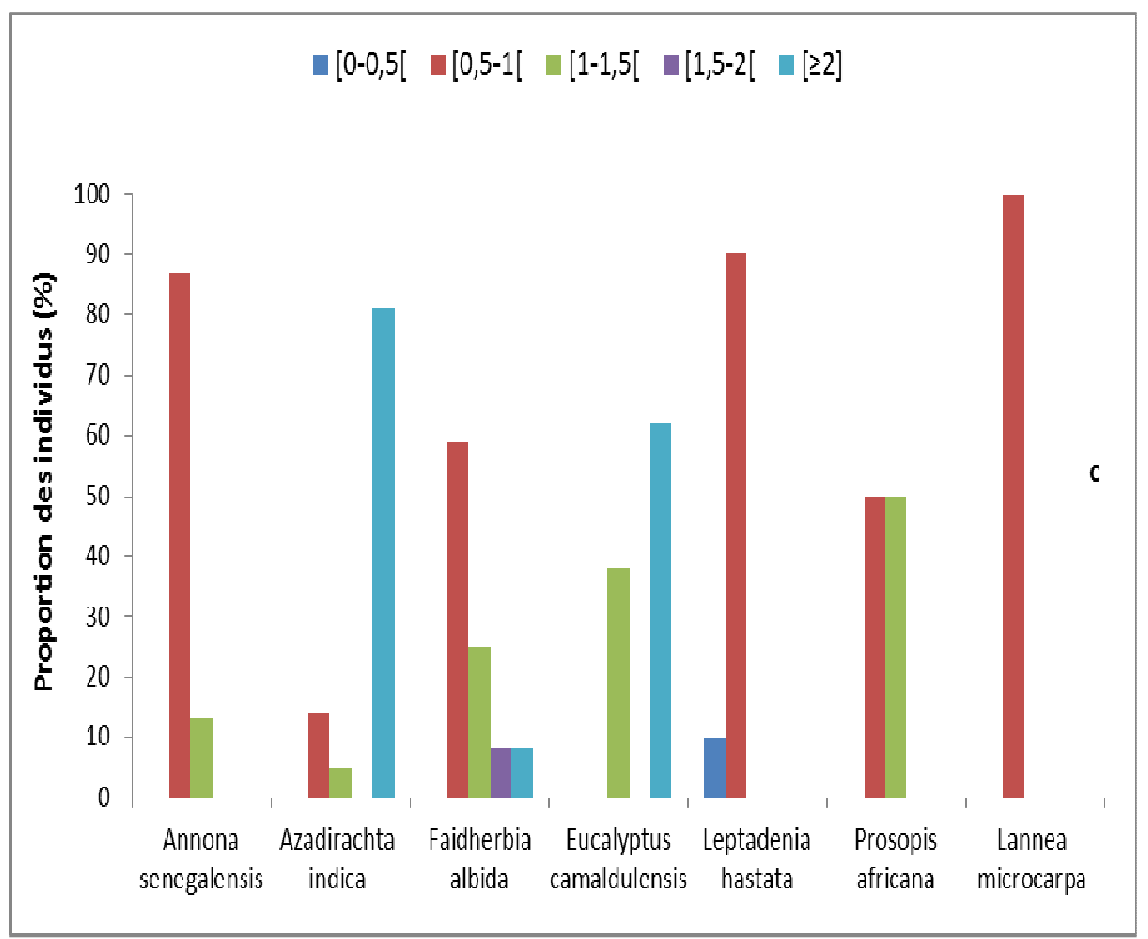

Figure 3c : Structure du peuplement juvénile des espèces dominantes à Daré. 


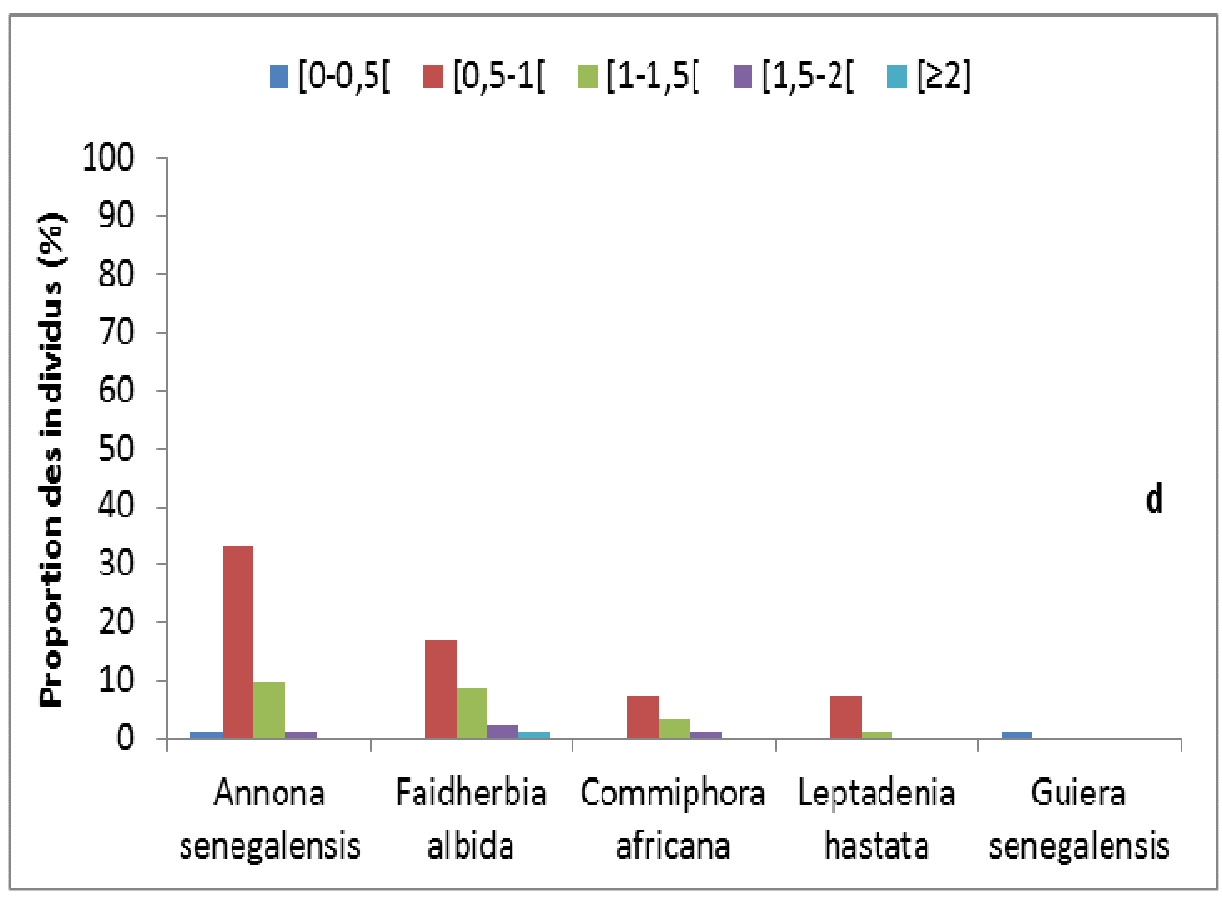

Figure 3d : Structure du peuplement juvénile des espèces dominantes à Zedrawa.

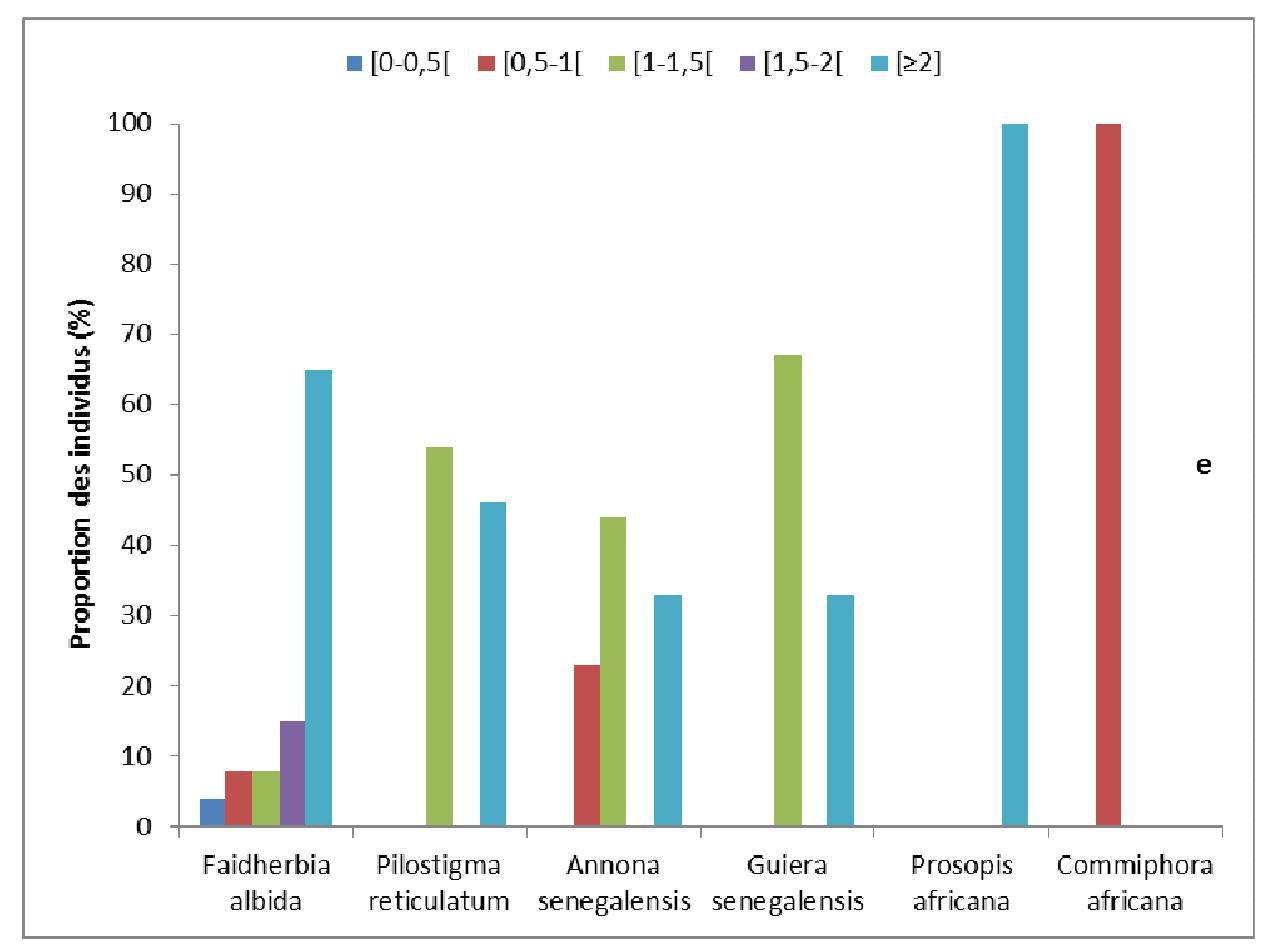

Figure 3e : Structure du peuplement juvénile des espèces dominantes à Ara Sofoua. 


\section{DISCUSSION}

Potentialités de la régénération ligneuse

La population juvénile dans les cinq sites d'études a montré une structure caractéristique d'une dynamique instable. Ce résultat est contraire à celui de Ouédraogo (2006) et Traoré (2012), qui ont remarqué que dans les parties orientale et occidentale du Burkina Faso, les structures de la population juvénile sont stables, caractéristique d'une bonne régénération. Ils ont cependant noté que la composition et la structure de la végétation ligneuse varient considérablement d'une localité à l'autre en fonction des facteurs environnementaux et des perturbations anthropiques.

\section{Régénération dans les sites de Maradi}

Hyphaene thebaica est très répandue dans la région de Maradi notamment dans le Goulbi N'kaba où il s'est imposé comme espèce agroforestière très appréciée par les paysans. Régis et al. (2008) affirment que la régénération naturelle assistée (RNA) peut permettre aux agriculteurs de reconstituer durablement un parc agroforestier à Hyphaene thebaica dans le Goulbi N'kaba. La structure des populations juvéniles de Guiera senegalensis et Combretum glutinosum est caractérisée par un important taux de mortalité dans la deuxième strate [0,5-1 m [. Mais, leur régénération est importante dans l'ensemble. Ces espèces ont une capacité de drageonnage relativement bonne et ce mode de reproduction asexuée peut justifier la structure démographique des populations juvéniles.

Hyphaene thebaica et Ziziphus mauritiana caractérisent la strate juvénile du site plus au Sud de la région de Maradi (El Guiéza) alors qu'au Nord (Dan Saga), c'est Guiera senegalensis et Combretum glutinosum qui dominent. Chaque espèce végétale est distribuée en fonction de sa propre tolérance aux multitudes des facteurs que comprend son environnement (Tremblay et al., 2002).

\section{Régénération dans les sites de Zinder}

Seul le site de Ara Sofoua présente une assez bonne régénération bien qu'il cache l'état réel de la régénération. L'absence d'individus dans la première classe [0-0,5 m [, à Daré et Zedrawa, traduit la pression des agriculteurs sur la régénération. En effet, les plantules de certaines espèces comme Annona senegalensis, Piliostigma reticulatum et Guiera senegalensis sont systématiquement coupées au ras du sol pour éviter l'effet de compétions avec les cultures. Cet état de fait a été évoqué par Baggnian et al., (2013). Cette pression se ressent dans la structure des juvéniles de ces espèces qui montrent un faible nombre d'individus dans les classes supérieures, traduisant l'inexistence de passerelle entre la population juvénile et les adultes. La sélection des espèces par les agriculteurs est guidée par leur capacité à régénérer et leur utilité en termes d'usages et de prestation de services (Akpo et al., 2003). Ouédraogo (2006) affirme qu'en milieu anthropisé, caractérisé par un système d'exploitation extensif des terres, l'avenir de certaines espèces dépend des types d'entretien ou protection.

Dans la région de Zinder, Faidherbia albida est la seule espèce à avoir une assez bonne aptitude de régénération. Cette espèce est protégée et épargnée de toute forme d'exploitation anarchique parce qu'elle présente certaines qualités aux yeux de la population. En effet, Faidherbia albida fournit du fourrage pour le bétail ou surtout procurent des revenus pour les paysans à travers la vente des gousses. Les rôles de certaines espèces dans la restauration de l'équilibre écologique et la vie sociale et économique leur confèrent un privilège de 
préservation délibérée (Abdoulaye et Ibro, 2006).

\section{Vitalité des peuplements}

La végétation endogène subit en général plus les effets de la pression anthropique. Les usages pour lesquels les prélèvements causent le plus de dommages aux individus sont le fourrage, la pharmacopée et l'alimentation humaine et animale. Les principales espèces concernées sont Commiphora africana, Guiera senegalensis, Prosopis africana, Sclerocarya birrea, Faidherbia albida, Lannea microcarpa, Ziziphus spina-christi. Les branches de Commiphora africana, Guiera senegalensis et Sclerocarya birrea sont utilisées pour le fourrage des bétails en général en début de saison de pluie (JuinAout). Prosopis africana issue de rejets des souches se casse très facilement à la suite de vents violents qui se manifestent généralement en début de la saison des pluies. Ces prélèvements constituent une menace pour la survie de nombreuses espèces utilitaires (Hahn-Hadjali et Thiombiano, 2000). Il faut noter que le degré de prélèvement varie suivant les espèces et celles-ci subissent une pression plus ou moins forte en fonction des localités.

\section{Dynamique de la végétation juvénile}

Les potentialités de régénération des ligneux reposent surtout sur le taux de survie des plants au cours de leur phase de développement. Suivant la structure démographique des juvéniles, on peut noter trois (3) principales phases (Ouédraogo et al., 2009). Dans la phase initiale, il y a un établissement et un bon développement des juvéniles qui a lieu dans la classe de [0-0,5 $\mathrm{m}[$. La seconde phase, qui est la plus critique, est caractérisée par un taux de survie très faible des populations qui se produit au cours du passage de la strate [0-0,5 m [ à [0,5-1 m [. La troisième phase de dynamique se caractérise par la stabilisation du taux de survie à partir de la strate [0,5-1 m [aux strates supérieures. Le recrutement massif des juvéniles dans la première strate est favorisé par les conditions hydriques du sol (Higgins et al., 2000) qui deviennent favorables avec l'installation de la saison pluvieuse. Le passage de la strate 1 à la strate 2 est marqué par un taux de mortalité supérieur à $30 \%$ dans certains cas. Cet état de fait s'explique par des facteurs perturbateurs. En effet, la récolte intensive des fruits ou des graines par exemple, peut entraîner une diminution progressive des ligneux qui les produit. Ce phénomène est observé pour Prosopis africana où la récolte excessive des gousses a sérieusement réduit la régénération naturelle dans les régions sahéliennes de l'Afrique de l'Ouest (Weber et al., 2008). Aussi, la pression anthropique a été évoquée par Baggnian et al. (2013), Baggnian et al. (2012), et Larwanou et al. (2010) qui affirment que le succès de restauration de la végétation dans la bande sud Niger est freiné par l'action de coupe frauduleuse occasionnée par les éleveurs transhumants et l'écorçage perpétré par certaines catégories de personnes, dont l'exploitation abusive rend certaines espèces vulnérables.

A cela s'ajoute des perturbations environnementales. Les processus de régénération des arbres peuvent être influencés par certains facteurs tels que le mode de dissémination, la viabilité, la dormance et la prédation des semences (Khurana et Singh, 2001), le stress hydrique, la structure du sol, la température, les attaques des insectes, le pâturage qui peuvent retarder le passage du stade juvénile au stade arbuste (Bationo et al., 2001). Des facteurs biotiques tels que la compétition intra- et interspécifique (pour la disponibilité en eau, en nutriments et 
en espace) peuvent aussi influencer la dynamique de la régénération naturelle (Dembélé et al., 2006).

En somme, le potentiel de régénération, en termes de levées naturelles, existe dans tous les terroirs villageois étudiés mais cela est meilleur dans la région de Maradi disposant d'un comité de surveillance de la RNA. Cela pourrait suggérer que les conditions sont plus favorables à l'établissement de la population des juvéniles. Les variations dans la densité des juvéniles pourraient être liées à l'interaction complexe entre des facteurs impliquant les caractéristiques des espèces, le type de sol, les conditions climatiques et les mécanismes de régénération des semences dans des stocks semenciers du sol, ainsi que la capacité de l'espèce à disposer de rejet de souches (Traoré, 2012). Dans le Centre-Sud Zinder, Faidherbia albida, malgré sa structure juvénile déséquilibrée, continuera à émerger ultérieurement comme principale espèce dominante dans la strate juvénile et adulte du fait qu'elle est la seule espèce protégée et épargnée de toute forme d'exploitation anarchique.

\section{Conclusion}

Dans le Centre-Sud du Niger, la pratique de la RNA est une activité bien connue des populations et est surtout focalisée sur des espèces les plus abondantes telles que Faidherbia albida, Combretum glutinosum, Guiera senegalensis Hyphaene thebaica et Piliostigma reticulatum. Cependant, dans la zone septentrionale de Maradi Guiera senegalensis et Combretum glutinosum montrent des signes de mauvais état. Dans le Sud Maradi, Hyphaene thebaica et Piliostigma reticulatum montrent une vulnérabilité des juvéniles. Dans le CentreSud Zinder, seul Faidherbia albida semblent présenter une structure des juvéniles stable. Le choix des espèces à protéger ainsi que la récurrence des facteurs de dégradation sont les principales causes de ce déséquilibre écologique. Cette situation met la végétation dans une dynamique fragile où d'importants changements floristiques peuvent survenir à court ou moyen terme. Ainsi beaucoup d'espèces sont rares et la pression qui s'exerce sur les individus juvéniles conduit inexorablement à leur disparition.

\section{REMERCIEMENTS}

Les auteurs remercient le Centre de Résilience de l'Université de Stockholm, pour avoir financé ces travaux ainsi que, la Faculté d'Agronomie de l'Université Abdou Moumouni de Niamey et l'Université de Maradi pour leur soutien multiforme. Nos remerciements vont aussi aux vaillantes populations des cinq villages.

\section{REFERENCES}

Abdoulaye T, Ibro G. 2006. Analyse des impacts socio-économiques des investissements dans la gestion des ressources naturelles: Étude de cas dans les régions de Maradi, Tahoua et Tillabéry au Niger. Etude Sahélienne, CRESA/Niamey, 65p.

Agnew CT, Chappell A. 1999. Drought in Sahel. Geo Journal, 48: 299-311.

Akpo LE, Banoin M, Grouzis M. 2003. Effet de l'arbre sur la production et la qualité fourragères de la végétation herbacée : Bilan pastoral en milieu sahélien. Méd. Vét., 154(10): 619-628.

Baggnian I, Adamou MM., Toudou A, Mahamane A. 2013. Impact du mode de gestion de la Régénération Naturelle Assistée des ligneux (RNA) sur la résilience des écosystèmes dans le centresud du Niger. Journal of Applied Biosciences, 71: 5742- 5752. 
Baggnian I, Mohamadou A, Adamou MM, Lawali S, Adam T, Enfors E, Larwanou M, Tougiani A. 2012. Perceptions paysannes des tendances du reverdissement des zones dégradées au Niger. Université de Maradi. Journal des Sciences de l'Environnement, 1(1): 43-52.

Bationo BA, Ouédraogo SJ, Alexandre DY, Guinko S. 2001. Statut hydrique de quatre espèces ligneuses soudaniennes dans la forêt de Nazinon, Burkina Faso. Sécheresse, 12(2): 87-94.

Bégué A, Vintrou E, Ruelland D, Claden M, Dessay N. 2011. Can a 25-year trend in Soudano-Sahelian vegetation dynamics be interpreted in terms of land use change? A remote sensing approach. Global Environmental Change, 21(2): 413-420.

Dembélé F, Picard N, Karembé M, Birnbaum P. 2006. Tree vegetation patterns along a gradient of human disturbance in the Sahelian areas of Mali. Journal of Arid Environment, 64: 284-297.

Dramé Y, Berti F. 2008. Les enjeux socioéconomiques autour de l'agroforesterie villageoise à Aguié (Niger). Tropicultura, 26(3): 141-149.

Giannini A, Biasutti M, Verstraete MM. 2008. A climate model-based review of drought in the Sahel: Desertification, the regreening and climate change. Global and Planetary Change, 64(3-4): 119-128.

Haglund E, Ndjeunga J, Snook L, Pasternak D. 2011. Dry land tree management for improved household livelihoods: farmer managed natural regeneration in Niger. Journal of Environmental Management, 92(7): 1696-705.

Hahn-Hadjali K, Thiombiano A. 2000. Perception des espèces en voie de disparition en milieu Gourmantché (Est $\mathrm{du}$ Burkina Faso). Berichte des
Sonderforshungsbereich, 268(14): 285297.

Herrmann S, Anyamba A, Tucker CJ. 2005. Recent trends in vegetation dynamics in the African Sahel and their relationship to climate. Global Environmental Change, 15(4): 394-404.

Higgins SI, Bond WJ, Trollope WSW. 2000. Fire, resprouting and variability: a recipe for grass-tree coexistence in savanna. $J$. Ecol., 88(2): 213-229.

Khurana E, Singh JS. 2001. Ecology of tree seed and seedling: implications for tropical forest conservation and restoration. Current Science, 80: 748-757.

Larwanou M, Dan Guimbo I, Oscar EM, Issaka AI. 2012. Farmer managed tree natural regeneration and diversity in a Sahelian environment: case study of Maradi region, Niger. Continental J. Agricultural Science, 6(3): 38 - 49.

Larwanou M, Saadou M. 2011. The role of human interventions in tree dynamics and environmental rehabilitation in the Sahel zone of Niger, Journal of Arid Environments, 75(2): 194-200.

Larwanou M, Saadou M. 2012. Impacts des activités de restauration des terres sur la végétation au Niger. Journal des Sciences de l'Environnement, 1(1): 1-15.

Mahamane A, Saadou M. 2008. Méthodes d'étude et d'analyse de la flore et de la végétation tropicale. Actes de l'atelier sur l'harmonisation des méthodes. Sustainable Use of Natural vegetation in West Africa, 78p.

Ouédraogo A. 2006. Diversité et Dynamique de la végétation ligneuse de la partie orientale du Burkina Faso. Thèse, Université de Ouagadougou, Burkina Faso, 230p.

Ouédraogo O, Thiombiano A, Hahn-Hadjali K, Guinko S. 2009. Diversité et dynamique de la végétation ligneuse 
juvénile du Parc National d'Arly (Burkina Faso). Candollea, 64: 257-278.

Régis P, Claudine SD, Aboubacar I. 2008. Valoriser les produits du palmier doum pour gérer durablement le système agroforestier d'une vallée sahélienne du Niger et éviter sa désertification. VertigOla Revue Electronique en Sciences de l'Environnement, $\quad \mathbf{8}(1)$. http://vertigo. revues.org/1452; DOI : 10.4000/vertigo. 1452.

Reij CP, Botoni E. 2009. La transformation silencieuse de l'environnement et des systèmes. Impacts des investissements publics et privés dans la gestion des ressources naturelles. CILSS, CIS, 63p.

Reistma JM. 1988. Forest Vegetation in Gabon. Tropenbos Technical Series 1. Tropenbos Foundation: The Netherlands; $142 \mathrm{p}$.

Sendzimir J, Reij CP, Magnuszewski P. 2011. Rebuilding Resilience in the Sahel: Regreening in the Maradi and Zinder Regions of Niger. Ecology and Society, 16(3): 1 .

Tougiani A, Guero C, Rinaudo T. 2009. Community mobilisation for improved livelihoods through tree crop management in Niger. Geo Journal, 74(5) : 377-389.

Toutain B. 1999. Dynamique de la végétation et évolution des écosystèmes pâturés au Sahel, the Sahel. SEREN Occasional Papers, 10 :1-11.

Traoré L, Ouédraogo I, Ouédraogo A, Thiombiano A. 2011. Perceptions, usages et vulnérabilité des ressources végétales ligneuses dans le Sud-Ouest du Burkina Faso. Int. J. Biol. Chem. Sci., 5(1): 258278.

Traoré L. 2012. Influence du climat et de la protection sur la végétation ligneuse de la partie Occidentale du Burkina Faso. Thèse de Doctorat unique, Université de Ouagadougou, 228p.

Weber JC, Larwanou M, Abasse TA, Kalinganire A. 2008. Growth and survival of Prosopis africana provenances tested in Niger and related to rainfall gradients in the West African Sahel. Forest Ecology and Management, 256(4): 585-592. 